\title{
A Comprehensive IVF laboratory COVID-19 Pandemic Response Plan
}

Fadi Choucair ${ }^{1,2 *}$; Nagham Younis ${ }^{1,3} ;$ Alia Hourani $i^{1,4}$

${ }^{1}$ Middle East Fertility Society Embryology Specialty Interest Group

${ }^{2}$ American University of Beirut Medical Center, Reproductive Endocrinology and Infertility unit. Email: fc13@aub.edu.Ib

${ }^{3}$ University of Jordan. Email: naghamyounis@yahoo.com

${ }^{4}$ Quttainah Medical Center, Kuwait. Email: aliahourani@gmail.com

\section{Abstract \\ Background}

The potential of the COVID-19 severe pandemic necessitates the development of an organized, rational plan for the operation of embryology/andrology laboratories without the compromise of the safety of personnel and gametes/embryos.

Main body

A comprehensive pandemic response plan was proposed for embryology and andrology laboratories for prepandemic preparedness, reduction or hibernation of procedures, and return to normal function. Preparation involves many plans and logistics before a pandemic risk rises. Many operational changes can be considered based on the severity and duration of the pandemic. This plan includes logistical arrangements, reducing labor needs, conserving supplies, protective measures for embryologists, and biological materials.

\section{Conclusion}

The sudden and unpredictable emergence of the COVID-19 pandemic dictate the need for preparedness plans for embryology/andrology laboratories as well as action-oriented plans to secure safety of gametes/ embryos and personnel.

Keywords: COVID-19; pandemic; response plan; in vitro fertilization laboratory; clinical embryology; andrology; emergency. 


\section{Background}

The recent onset of a worldwide COVID-19 pandemic brings to bear the need for disaster plans of IVF laboratories. Many experts have posited modern-day pandemics inevitable. Given that an estimated of more than $25 \%$ of transmissions are believed to occur in the workplace [1].

The reproductive societies, such as American Society for Reproductive Medicine (ASRM) and the European Society for Human Reproduction and Embryology (ESHRE), have strongly urged to stop the main functions during COVID-19 pandemic mainly for the protection of human life (i.e., conceptus); however, they did not provide specific practical guidance for the laboratories during a pandemic event and after the resolution of the risk.

To meet this challenge, we suggested a Response Plan to outline essential services and proposed the means to maintain these services in the event of a severe pandemic. To that end, the Middle East Fertility Society Embryology specialty interest group provided practical recommendations for a comprehensive preparedness emergency plan for the protection of human life (gametes/embryos and staff), protection of assets, and proposed practices for the return to normal function phase of andrology/embryology laboratories.

\section{Main text}

\section{Heightened planning and logistic preparation. Initial onset of a pandemic}

The majority of prepandemic planning occurs at an early level, as outlined in Figure 1 and discussed later. Level zero (no risk of a pandemic emergence) response plan primarily entails that embryologists should be educated about handwashing with soap and water and the use of embryo-tested hand disinfectants before entering the laboratories. Hand hygiene compliance observation audits should be periodically performed [2]. Standard operating procedure (SOP) for Hand Hygiene Observation Audit should also be prepared with stress on avoidance of touching noses, mouths, and eyes. Embryologists should obtain a vaccination, and they should participate in a fit testing for N95 masks.

Personnel should know whom they are to contact during an emergency [3]. Along this perspective, the laboratory supervisor should prepare staff emergency and suppliers contact lists that can be accessible for all staff.

As for the laboratory assets, a spare storage cryotank and sufficient liquid nitrogen (LN2) supply to fill should be maintained on-site (College of American Pathologists CAP accreditation 2019 checklist; RLM.03944). The cryotanks need to be periodically decontaminated using laboratory detergents and rinsed in distilled water [4]. It is important to stress that all storage tanks should be filled at least weekly wearing 
personal protective equipment (PPE) such as insulated thermal gloves and protective eyewear [5]. Cryopreservation consents prepared should state the possibility of failures during storage in case of disasters [3].

Duplicate records are maintained on a secure web server and updated regularly [3].

Additionally, facilities must consider storing cleaned spare gas cylinders in a ventilated storage room due to potential errors in the delivery [6].

As pandemic risks increase (moderate risk of pandemic emergence), more stringent social distancing techniques and the use of personal protective equipment are recommended. Employees are educated to recognize early signs of illness, such as sore throat, fever, and cough, and staying home when sick.

Laboratory manager identifies essential roles such as embryologists performing oocyte vitrification. He may contact free-lancers to fill essential roles. In addition, the managers calculate essential PPE supply as well as laboratory supplies needed. A supply storage plan is developed, and alternative culture media batches with longer expiration dates are secured. At this phase, the laboratory manager initiates discussions with supply distributors.

- Develop plans for later pandemic stages

- Educate staff on disease transmission mitigation methods

- Perform hand hygiene compliance observation audits

- Prepare staff emergency and suppliers contact lists

- Participate in a fit testing for N95 masks

- Identify essential roles in the laboratory (e.g., vitrification operators) and assign back up personnel

- Dedicate a spare storage cryotank and sufficient liquid nitrogen supply

- Stress on using PPE and protective eyewear during liquid nitrogen filling

- Update and maintain records on a remote server

- Store cleaned spare gas cylinder

- Calculate essential personnel supply needs

- Develop supply storage plan (media with longer expiration dates, cryopreservation medium stock)

- Initiate discussion with supply distributors

Figure 1. Early-levels response plan (no risk or moderate risk of pandemic emergence): recommendations for embryology/andrology laboratories assessment and pre-pandemic planning.

\section{Reduction or hibernation of procedures. Later pandemic stages}

Further pandemic progression (pandemic imminent and pandemic period) may sufficiently reduce the number of procedures performed. In extreme scenarios, the 
clinic may elect to cease all procedures. Multiple operational practices can be changed to adapt to the pandemic risk (Figure 2).

\section{Operational changes reducing labor needs and conserving supplies.}

The number of embryologists reporting to work can be reduced. Embryologists would be expected to perform rotating shifts of 2 weeks each. The rotating schedule is created to minimize exposure time to infection while accommodating the continuity of care within the laboratory. During a severe pandemic, manufacturing distributing companies may lack the personnel to maintain a regular delivery schedule. This shortage would require the laboratory to increase its stock mainly of PPE (gloves, surgical masks) and cryopreservation media/devices. Similarly, supplies inventory is updated regularly and accessible to infer the needs during the pandemic and the back to normal function phase.

Besides that, workload reduction requires other considerations of resources managements such as reduction of the number of operated incubators.

\section{Operational changes protecting embryologists and gametes/embryos}

It is rational that patients should be screened, and staff are regularly tested. In addition, several strict practices should be emphasized and implemented to protect the personnel and the gametes/embryos. In this regard, proper PPE should be worn by all staff namely face mask, nitrile powder-free gloves used at all steps, and impermeable gowns over the scrub.

In addition, air quality filters and positive pressure need to be checked. A thorough cleaning of supply boxes and gas cylinders practices before entering the laboratory should be emphasized using routine laboratory disinfection agents. Ideally, supplies should be received away from the laboratory. The biosafety cabinet workspace is thoroughly disinfected and uncluttered to avoid flow turbulence. Along this perspective, personnel should exercise laboratory housekeeping vigilance during high pandemic risk. Cleaning practices can be performed by laboratory personnel, such as disinfecting common surfaces (e.g., doorknobs, keyboards, surfaces...). Ultraviolet lamps in biological safety cabinets should be left on after duty hours.

It is rational to reduce unnecessary patient visits such as visits for cryopreservation storage renewal payment and send cryopreservation consents to patients through email prior to signature. It is essential for embryologists to wear proper PPE (N95 masks and protective eyewear) when dealing with patients or communicating information.

Coronavirus was detected in the blood of infected patients [7]; thus, special precautions should be taken in handling specimens and the preparation (follicular 
fluid dishes, semen ejaculate jars, testicular tissue processing) of which involves steps that can lead to aerosol formation. It appears prudent that special consideration should be applied during oocyte pick up in a way that minimizes splashes in addition to the use of universal precautions. Cumulus oocyte complexes should be thoroughly washed to dilute viral titer. More so, a large piece of absorbent material can be placed at the floor in case of blood spillage and collected follicular fluid can be poured into a sterile cup before disposal.

Basic science studies identified the presence of type 2 Angiotensin-converting enzymes (ACE2) receptors, which mediate the entry of coronavirus, on human spermatozoa [8]. Furthermore, ACE2 receptors were also found expressed on Sertoli and Leydig cells [9]. Thus, it would be rational to be cautious while processing semen and testicular tissue. However, very recent studies reported no evidence of transmission of 2019-nCov through sperm [10,11].

Preparatory steps that may generate aerosols or droplets include expelling aspirates, and mechanical processing of testicular tubules may be performed using appropriate PPE including gloves, and goggles or face shields. It should be emphasized the frequent change of sterile Pasteur pipettes and tubes prior to sperm washing procedures [12]. Sperm density gradient technique can be followed by a swim-up step if sperm is satisfactory. It is advised to use sterile-filtered tips pipettes and to regularly UV disinfect micropipettes or disassemble the devices then disinfect using regular laboratory agents.

Regarding cryopreservation, liquid nitrogen Styrofoam box should be disinfected before being filled with liquid nitrogen. Sterility of factory-derived LN2 often is not guaranteed, although the risk of contamination by human pathogens may theoretically exist. In theory, some air-borne contaminants can contaminate liquid nitrogen during the process of compressing nitrogen [4]. Practically, during cryopreservation, embryologists should wear goggles to avoid contact with liquid nitrogen vapor; if available, use UV sterilized liquid nitrogen; sequentially wash embryos or oocytes and examine the integrity of the zona pellucida. Zona pellucida is considered as an efficient barrier to viral contamination [13]. Ideally, a closed system for cryopreservation should be encouraged to secure safe storage.

It is noteworthy to mention that alarming findings from a very recent study of singlecell RNAseq datasets revealed that human embryos express the receptors for SARSCoV-2 and other coronaviruses [14]. Therefore, embryologists should take strict precautions or ideally frequently screened for SARS-CoV-2. 
Operational changes reducing labor needs and conserving supplies.

- Create rotation shifts for personnel

- Increase the stock of PPE

- Increase the stock of cryopreservation media/devices

- Update regularly supplies inventory

- Reduce the number of operated incubators

\section{Operational changes protecting embryologists and gametes/embryos}

- Screen patients and regularly screen staff

- Use proper PPE (face mask, nitrile powder-free gloves at all steps, and impermeable gowns over the scrub)

- Stress on cleaning gas cylinders and supplies boxes before entering the laboratory

- Biosafety cabinet workspace should be thoroughly disinfected and uncluttered

- Cleaning practices can be performed by laboratory staff (surfaces, keyboards, doorknobs...)

- Postpone visits for cryopreservation storage renewal payment

- Send cryopreservation consents and information to patients through email prior to signature

- Increasing vigilance on checking air quality filters and positive pressure

- Turn on the ultraviolet lamp in biological safety cabinets after duty hours

- Wear proper PPE when dealing with patients or communication information

- Minimize blood splashes during the oocyte pick up procedure (e.g., use large absorbent material on the floor; collect follicular fluid in a cup before disposal)

- Thoroughly wash cumulus-oocyte complexes

- Use protective eyewear during testicular tissue processing

- Emphasize the use of density gradient for sperm processing

- Stress on changing pipettes and tubes between sperm washing steps

- Use filtered tips and regularly UV disinfect micropipettes or disassemble the devices then disinfect using regular laboratory agents

- Disinfect liquid nitrogen Styrofoam box

- Use protective eyewear during handling LN2

- Sterilize LN2 with UV

- Sequentially wash oocytes or embryos

- Encourage the use of cryopreservation closed-systems

- Avoid breaching the integrity of the zona pellucida

Figure 2. Late pandemic stages (pandemic imminent and pandemic period) response plan: recommendations for embryology/andrology laboratories assessment and planning during reduction or hibernation of procedures. 


\section{Return to function (Figure 3)}

Once the pandemic illness has subsided, all facility areas will be disinfected thoroughly, such as incubators, biosafety cabinets, fridges, centrifuges [4], and cryotanks using the regular disinfectant used. Similarly, all unused equipment or spaces will be sanitized thoroughly, calibrated, and validated. Although these areas would be cleaned during hibernation, the reduced activity might lead to the build-up of dust or other fomites such as gas and supplies storage rooms. All essential personnel would be provided time-off if the clinic continued its services during the pandemic period. A best practice would be to regularly screen all patients and personnel. Unless the World Health Organization changes the status of the pandemic, all protective precautions (Figure 2) during the pandemic stage are encouraged to be followed.

- Screen patients and regularly screen staff

- Disinfect all facility areas (incubators, biosafety cabinets, fridges, centrifuges, and cryotanks)

- Clean unused areas (gas room, storage supply room...)

- Initiate discussions with supply distributors

- Provide time-off to essential personnel

- Follow strict protective measures and implement operational changes until the WHO changes the pandemic status

Figure 3. Late pandemic stages response plan: recommendations for embryology/andrology laboratories assessment and planning during return to normal function phase

\section{Conclusion}

Planning for a crisis is one of the most important undertakings of a laboratory. Appropriate planning protects both personnel and gametes/embryos and allows for continuity of care. The planning process involves the identification of risks and operational changes to address staffing, supply reductions, and laboratory practices. 


\section{List of abbreviations}

COVID-19: coronavirus disease 2019

IVF: in vitro fertilization

ASRM: American Society for Reproductive Medicine

ESHRE: European Society for Human Reproduction and Embryology

SOP: standard operating procedure

LN2: liquid nitrogen

CAP: College of American Pathologists

PPE: personal protective equipment

ACE2: type 2 Angiotensin-converting enzymes

2019-nCov: novel coronavirus

UV: ultraviolet

SARS-CoV-2: severe acute respiratory syndrome coronavirus 2

RNAseq: RNA sequencing

WHO: World Health Organization

Declarations

\section{Ethics approval and consent to participate}

Not applicable.

\section{Consent for publication}

Not applicable.

\section{Availability of data and materials}

Not applicable

\section{Competing interests}

The authors declare that they have no competing interests.

\section{Funding}

None.

\section{Authors' contributions}

FC wrote the manuscript. NY critically revised the article. FC, NY, and $\mathrm{AH}$ provided the current recommendations.

Acknowledgments: This report was developed under the direction of the Middle East Fertility Society Embryology Specialty Interest Group as a service to embryologists and laboratory technicians. While this document reflects the appropriate management of a pandemic encountered in the practice of clinical embryology, it is not intended to be a standard of practice or to dictate an exclusive plan but rational recommendations for practice during a pandemic. 
The following members of the MEFS Embryology SIG executive team participated in the development of this document: Fadi Choucair, Ph.D.; Nagham Younis, M.Sc; Alia Hourani, M.Sc.

\section{References}

1. Koh D. (2020). Occupational risks for COVID-19 infection. Occupational Medicine (Oxford, England) 70:3.

2. Health NIf and C Excellence. (2014). Infection prevention and control. Quality standard QS61.

3. Technology PCotSfAR and PCotASfR Medicine. (2008). Guidelines for development of an emergency plan for in vitro fertilization programs. Fertility and sterility 90:S131S133.

4. Bielanski A and G Vajta. (2009). Risk of contamination of germplasm during cryopreservation and cryobanking in IVF units. Human reproduction 24:2457-2467.

5. Schiewe M, M Freeman, J Whitney, M VerMilyea, A Jones, M Aguirre, C Leisinger, G Adaniya, N Synder and R Chilton. (2019). Comprehensive assessment of cryogenic storage risk and quality management concerns: best practice guidelines for ART labs. Journal of assisted reproduction and genetics 36:5-14.

6. Elder K, M Van den Bergh and B Woodward. Troubleshooting and Problem-solving in the IVF Laboratory. (2015). Cambridge University Press.

7. Ng LF, M Wong, S Koh, E-E Ooi, K-F Tang, H-N Leong, A-E Ling, LV Agathe, J Tan and ET Liu. (2004). Detection of severe acute respiratory syndrome coronavirus in blood of infected patients. Journal of clinical microbiology 42:347-350.

8. Köhn FM, C Müller, D Drescher, C Neukamm, KE Mulla, R Henkel, W Hägele, E Hinsch, $U$ Habenicht and WB Schill. (1998). Effect of angiotensin converting enzyme (ACE) and angiotensins on human sperm functions. Andrologia 30:207-215.

9. Fan C, K Li, Y Ding, WL Lu and J Wang. (2020). ACE2 expression in kidney and testis may cause kidney and testis damage after 2019-nCoV infection. MedRxiv.

10. Song C, Y Wang, W Li, B Hu, G Chen, P Xia, W Wang, C Li, F Diao and Z Hu. (2020). Absence of 2019 Novel Coronavirus in Semen and Testes of COVID-19 Patients. Biology of Reproduction.

11. Pan F, X Xiao, J Guo, Y Song, H Li, DP Patel, AM Spivak, PA Joseph, X Zhang and C Xiong. (2020). No evidence of SARS-CoV-2 in semen of males recovering from COVID-19. Fertility and Sterility.

12. Nicholson C, L Abramsson, S Holm and E Bjurulf. (2000). Bacterial contamination and sperm recovery after semen preparation by density gradient centrifugation using silane-coated silica particles at different $g$ forces. Human Reproduction 15:662-666.

13. Van Soom A, A Wrathall, A Herrler and H Nauwynck. (2009). Is the zona pellucida an efficient barrier to viral infection? Reproduction, fertility and development 22:21-31.

14. Colaco S, K Chhabria, N Singh, A Bhide, D Singh, A Singh, A Husein, A Mishra, R Sharma and N Ashary. (2020). Expression of SARS-CoV-2 receptor ACE2 and the spike protein processing enzymes in developing human embryos. arXiv preprint arXiv:2004.04935. 